\title{
Testosterone treatment for men with chronic heart failure
}

\author{
P J Pugh, R D Jones, J N West, T H Jones, K S Channer
}

Heart 2004;90:446-447. doi: 10.1136/ hrt.2003.014639

C hronic heart failure (CHF) is a disabling disease characterised by exercise intolerance and dyspnoea. Disease progression arises from prolonged neurohormonal and pro-inflammatory cytokine activation and is associated with a metabolic shift favouring catabolism, vasodilator incapacity, and loss of skeletal muscle bulk and function. In men, androgens are important determinants of anabolic function and physical strength. Androgens also possess anti-inflammatory and vasodilatory properties. In addition, testosterone has been shown to augment cardiac output acutely in men with CHF. ${ }^{12}$ Low plasma concentrations of testosterone have been described in men with CHF, and correlate positively with cardiac output. ${ }^{3}$ It was hypothesised that relative hypotestosteronaemia could contribute to clinical features of muscle wasting and exercise intolerance, inflammatory cytokine activation and impaired vasodilatation, and progression of heart failure. This pilot study sought to determine whether testosterone treatment could improve exercise capacity and symptoms in male patients with CHF.

\section{METHODS}

Twenty ambulant male patients (median age 62 years, range 44-81), with CHF of at least six months duration and no other malignant or debilitating disease, took part in a randomised, double blind, placebo controlled trial of testosterone (Sustanon 100, Organon Laboratories, Cambridge, UK) or placebo treatment ( $1 \mathrm{ml} 0.9 \%$ saline, Martindale Pharmaceuticals, Romford, UK) given by intramuscular injection every two weeks for 12 weeks. Informed consent was obtained and the study was approved by the local research ethics committee. Subjects had at least moderate impairment of left ventricular systolic function (mean (SD) ejection fraction $35(8) \%)$ and reduced exercise tolerance. Men were excluded if prostate specific antigen (PSA) concentration was high or exercise limitation was due to a non-cardiac cause. All were taking angiotensin converting enzyme (ACE) inhibitors and 80\% were also taking diuretics.

Subjects were assessed between $8.00 \mathrm{am}$ and $9.30 \mathrm{am}$ at baseline and then 12 weeks later. They completed a short questionnaire detailing medical history and resting heart rate; blood pressure and weight were measured. Exercise tolerance was assessed using the incremental shuttle walk test (SWT). ${ }^{4}$ This is a symptom limited test with a progressive increase in workload, highly reproducible after one practice test, which is designed to allow subjects to achieve maximum exercise duration. Each patient completed two SWTs one week apart before starting treatment, and the result of the second test was used for analysis.

Left ventricular ejection fraction was determined by transthoracic echocardiography using Simpson's rule. To estimate skeletal muscle bulk, transverse computed tomographic (CT) images of the mid-thigh and mid-calf of both legs were obtained and the cross sectional area of skeletal muscle at these points determined by planimetry. Physical strength was measured using a hand held grip dynamometer. Blood was taken for measurement of full blood count, sex hormones, PSA, brain natriuretic peptide (BNP), and

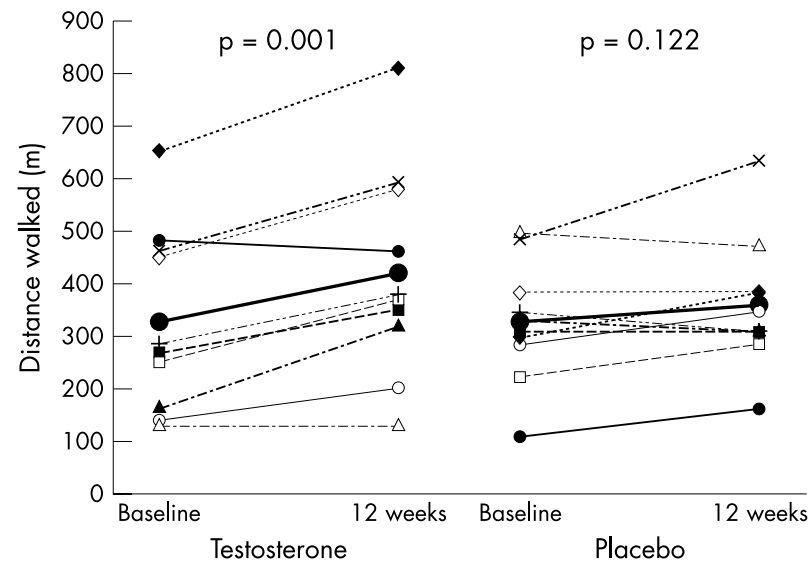

Figure 1 Changes in distance achieved in the shuttle walk test. Lines in bold with large solid circles indicate mean values in each group. Mean (SD) distance walked at baseline was similar in testosterone and placebo treated groups (328 (174) $\mathrm{m} v 314(92) \mathrm{m}, \mathrm{p}=0.825)$. In the testosterone treated group, exercise duration increased significantly to $419(200) \mathrm{m}, \mathrm{p}=0.001$, but there was no significant change with placebo, to $340(101) \mathrm{m}, \mathrm{p}=0.122$.

cytokines (TNF $\alpha$, Il-1 $\beta$, and Il-6). The Minnesota living with heart failure (MLHF) questionnaire was used for heart failure symptoms and quality of life and the Beck depression inventory (BDI) was used for assessment of depression.

Data were tested for normality using the KolmogorovSmirnov test and within group changes following treatment were analysed using the paired $t$ test for normal data and Wilcoxon matched pairs test for other data. Independent samples $t$ tests or Mann Whitney U test were used for between group comparisons ( $\mathrm{p}<0.05$ was considered significant).

\section{RESULTS}

Active and placebo groups were well matched with no significant differences at baseline for all measured parameters and concomitant treatment.

There was a significant increase in the distance walked in the SWT in subjects who received testosterone, but not in the placebo group (fig 1). The mean (SEM) increase in distance walked was significantly greater following testosterone treatment $(91.0 \quad(19.7) \mathrm{m} \quad v \quad 26.0 \quad(15.2) \mathrm{m}, \mathrm{p}=0.018)$, representing a treatment effect of $65.0 \quad(24.9) \mathrm{m} \quad(95 \%$ confidence interval (CI) 12.6 to 117.4). The mean (SEM) of all individual percentage changes from baseline was $32.9 \%$ (9.1) $v 10.0 \%$ (4.8), $\mathrm{p}=0.038$. There was no relation between baseline hormone values and response to treatment.

Abbreviations: $A C E$, angiotensin converting enzyme; $B D I$, Beck depression inventory; $B N P$, brain natriuretic peptide; CHF, chronic heart failure; $C T$, computed tomography; M LHF, Minnesota living with heart failure; PSA, prostate specific antigen; SW T, shuttle walk test 
MLHF symptom scores improved in the testosterone group (34.5 (29.3) to 26.5 (25.3), $\mathrm{p}=0.034)$, with a trend to lower BDI score $(7.3(7.3)$ to $5.7(6.0), p=0.053)$. Placebo had no effect on symptom scores. There was a trend to greater reduction in MLHF scores with testosterone $(-8.0$ (3.2) $v 1.3(4.0)$, $\mathrm{p}=0.086)$ but the reduction in BDI was not significantly different from placebo $(-1.6(0.7) v-1.5(0.9), \mathrm{p}=0.933)$.

Treatment had no significant effect on measurements of skeletal muscle bulk and strength, heart rate, blood pressure, weight, left ventricular size and function, QTc, nor plasma concentrations of cytokines. There was a trend to greater reduction in mean (SD) BNP value with testosterone treatment compared with placebo $(-9.3$ (17.6) $\mathrm{pg} / \mathrm{ml} v 4.5$ (10.9) $\mathrm{pg} / \mathrm{ml}, \mathrm{p}=0.063$ ).

One patient in the active group was admitted to hospital with breathlessness after eight weeks of treatment. He was discharged within 48 hours on a higher dose of diuretic and continued in the trial to completion without unblinding. No other patient complained of deterioration in symptoms and treatment remained constant. There was no significant change in plasma concentrations of PSA or in haematological parameters in either group.

\section{DISCUSSION}

We found significant improvements in exercise capacity and symptoms in men with heart failure following treatment with testosterone. The mechanism of benefit is unclear. The small sample size limited our ability to detect any significant impact of treatment on myocardial contractility, physical strength or humoral factors.

Alterations of skeletal muscle in heart failure patients include changes in muscle microstructure and cellular energy handling and it may be that microscopic, but not macroscopic, muscle changes contributed to improved exercise capacity and that these changes were undetectable by the imaging modality used. Also, the population studied was relatively unselected and it may be that subpopulations of patients with cytokine activation, cachexia, and sarcopenia may derive even greater benefit than that observed in this trial. Further studies are warranted in patients with more advanced disease.

A trend to positive effect of testosterone on mood scores was observed. CHF patients have higher depression scores than controls, as do testosterone deficient men, in whom replacement may reduce depressive symptoms. ${ }^{5}$ The improvement in mood may have been related to improved functional capacity, vice versa or an independent phenomenon.

In conclusion, in men with CHF, 12 weeks of treatment with testosterone was safe, well tolerated, and led to significant improvements in physical capacity and symptoms. The mechanism of action of testosterone and confirmation of its safety and beneficial effect in selected patients require further evaluation in larger clinical trials.

\section{ACKNOWLEDGEMENTS}

This trial was funded by a project grant from the National Heart Research Fund. The authors thank Dr Edwin van Beek for help with acquisition and interpretation of CT images.

\section{Authors' affiliations}

P J Pugh, J N West, K S Channer, Department of Cardiology, Royal Hallamshire Hospital, Sheffield, UK

R D Jones, T H Jones, Academic Unit of Endocrinology, Division of G enomic Medicine, University of Sheffield M edical School, Sheffield, UK

Correspondence to: Dr K S Channer, M131, Royal Hallamshire Hospital, Glossop Road, Sheffield, S10 2JF, UK;

Kevin.Channer@sth.nhs.uk

Accepted 30 June 2003

\section{REFERENCES}

1 Pugh PJ, Jones RD, Jones TH, et al. Heart failure as an inflammatory condition: potential role for androgens as immune modulators. Eur J Heart Failure 2002;4:673-80.

2 Pugh PJ, Jones TH, Channer KS. A cute haemodynamic effects of testosterone in men with chronic congestive heart failure. Eur Heart $J$ 2003;24:909-15.

3 Tappler B, Katz M. Pituitary-gonadal dysfunction in low-output cardiac failure. Clin Endocrinol 1979;10:219-26.

4 Singh SJ, Morgan M DL, Scott S, et al. Development of a shuttle walking test of disability in patients with chronic airways obstruction. Thorax 1992;47:1019-24

5 Wang C, A lexander G, Berman N, et al. Testosterone replacement therapy improves mood in hypogonadal men - a clinical research centre study. J Clin Endocrinol Metab 1995;81:3578-83.

\section{WEB TOP 10}

www.heartinl.com

These articles scored the most hits on Heart's website during January 2004

1 Clinical implications of the new definition of myocardial infarction

JK French, HD White

January 2004;90:99-106. (Education in Heart)

2 Masquerade: a malignant arrhythmia masquerading as benign

$R$ Nandakumar, JC Patel, P Broadhurst

February 2004;90:e10. (Case report)

3 Indications for the closure of patent foramen ovale MJ Landzberg, P Khairy

February 2004;90:219-24. (Education in Heart)

4 Is impairment of ischaemic preconditioning by sulfonylurea drugs clinically important?

JJ Meier, B Gallwitz, WE Schmidt, A Mugge, MA Nauck January 2004;90:9-12. (Review)

5 Implantable cardioverter-defibrillator treatment and resynchronisation in heart failure

$C$ Linde

February 2004;90:231-4. (Education in Heart)

6 Chronic therapeutically refractory angina pectoris

MJL DeJongste, RA Tio, RD Foreman

February 2004;89:225-30. (Education in Heart)

7 Fundamental concepts in myocardial viability assessment revisited: when knowing how much is "alive" is not enough RJ Kim, DJ Shah

February 2004;90:137-40. (Mini-symposium)

8 The role of echocardiography in atrial fibrillation and cardioversion

RW Troughton, CR Asher, AL Klein

December 2003;89:1447-54. (Education in Heart)

9 New tools for assessing microvascular obstruction in patients with ST elevation myocardial infarction

JA de Lemos, JJ Warner

February 2004;90:119-20. (Editorials)

10 Is Mobitz type I atrioventricular block benign in adults? DB Shaw, Jl Gowers, CA Kekwick, KHJ New, AWT Whistance

February 2004;90:169-74. (Cardiovascular medicine)

Visit the Heart website for hyperlinks to these articles, by clicking on "Top 10 papers"

www.heartinl.com 